\title{
Historein
}

Vol 4 (2003)

Public Histories

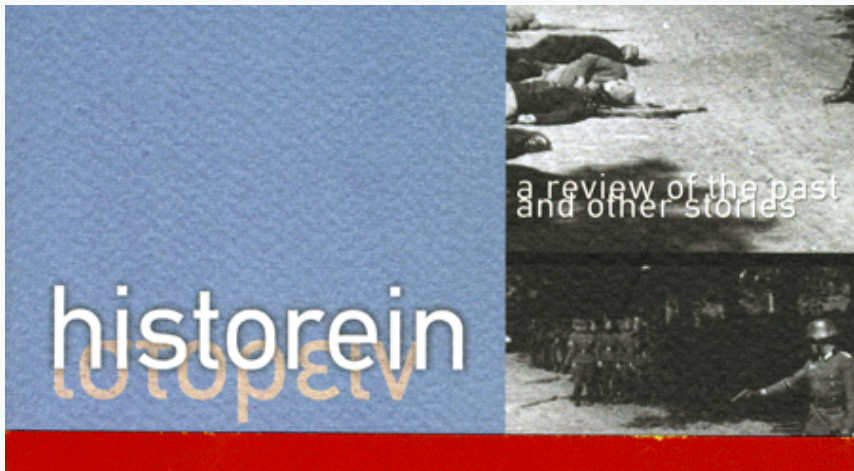

\section{Progrès et catastrophe. La conception de l'histoire} de Walter Benjamin

\section{Michael Löwy}

doi: $10.12681 /$ historein.88

\section{Copyright @ 2012, Michael Löwy}

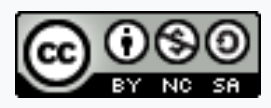

This work is licensed under a Creative Commons Attribution-NonCommercialShareAlike 4.0.

To cite this article:

Löwy, M. (2004). Progrès et catastrophe. La conception de l'histoire de Walter Benjamin. Historein, 4, 199-205. https://doi.org/10.12681/historein.88 


\section{INTERVENTIONS}

\section{Progrès et}

catastrophe.

La conception

de l'histoire

de Walter

Benjamin

de Michael Löwy
HISTOREIN

Nous sommes habitués à classer les différentes philosophies de l'histoire selon leur caractère progressiste ou conservateur, révolutionnaire ou nostalgique du passé. Walter Benjamin échappe à ces classifications. C'est un critique révolutionnaire de la philosophie du progrès, une adversaire marxiste du "progressisme", un nostalgique du passé qui rêve de l'avenir.

La réception de Benjamin, notamment en France et aux USA, s'est intéressé prioritairement pour le versant esthétique de son oeuvre, avec une certaine propension à le considérer surtout comme un historien de la culture ou un critique littéraire. Or, sans négliger cet aspect de son oeuvre, il faut mettre en évidence la portée beaucoup plus vaste de sa pensée, qui vise rien moins qu'une nouvelle compréhension de l'histoire humaine. Les écrits sur l'art ou la littérature ne peuvent être compris que par rapport à cette vision d'ensemble qui les illumine de l'intérieur.

La philosophie de l'histoire de W. Benjamin puise à trois sources très différentes: le romantisme allemand, le messianisme juif, le marxisme. Elle n'est pas une combinatoire ou "synthèse" de ces trois perspectives (apparemment) incompatibles, mais l'invention, à partir d'elles, d'une nouvelle conception, profondément originale.

L'expression "philosophie de l'histoire" risque d'induire en erreur. II n'y a pas, chez Benjamin, de système philosophique: toute sa réflexion prend la forme de l'essai ou du fragment - quand ce n'est pas de la citation pure et simple, les passages arrachés à leur contexte étant mis au service de sa démarche propre. Toute tentative de systématisation est donc problématique et incertaine. Les brèves remarques qui suivent ne sont que quelques pistes de recherche. 
On trouve souvent dans la littérature sur Benjamin deux erreurs symétriques qu'il faut éviter à tout prix: la première consiste à dissocier, par une opération (au sens clinique du terme) de "coupure épistémologique", l'oeuvre de jeunesse "idéaliste" et théologique de celle, "matérialiste" et révolutionnaire, de la maturité; la deuxième, par contre, envisage son oeuvre comme un tout homogène et ne prend nullement en considération le bouleversement profond apporté, vers le milieu des années 20, par la découverte du marxisme. Pour comprendre le mouvement de sa pensée il faut donc considérer simultanément la continuité de certains thèmes essentiels, et les divers tournants et ruptures qui jalonnent sa trajectoire intellectuelle et politique.

On pourrait prendre comme point de départ la conférence de 1914 sur "La vie des étudiants", qui présente, d'emblée, quelques unes des principales lignes de force de cette trajectoire. Les remarques qui ouvrent cet essai contiennent une amorce surprenante de sa philosophie messianique de l'histoire:

"Confiante en l'infini du temps, une certaine conception de l'histoire discerne seulement le rythme plus ou moins rapide selon lequel hommes et époques avancent sur la voie du progrès. D'où le caractère incohérent, imprécis, sans rigueur, de l'exigence adressée au présent. Ici, au contraire, comme l'ont toujours fait les penseurs en présentant des images utopiques, nous allons considérer l'histoire à la lumière d'une situation déterminée qui la résume comme en un point focal. Les éléments de la situation finale ne se présentent pas comme informe tendance progressiste, mais, à titre de créations et idées en très grand péril, hautement décriées et moquées, ils s'incorporent de façon profonde en tout présent. (...) Cette situation (...) n'est saisissable que dans sa structure métaphysique, comme le royaume messianique ou comme l'idée révolutionnaire au sens de 89." 1

Les images utopiques - messianiques et révolutionnaires - contre l' informe tendance progressiste: voici posés, en raccourci, les termes du débat que Benjamin va poursuivre à travers toute son oeuvre. Comment cette première intuition va-t-elle s'articuler plus tard avec le matérialisme historique ?

C'est à partir de 1924, quand il lit Histoire et Conscience de Classe de Lukacs - et découvre le communisme au travers les yeux d'Asja Lacis - que le marxisme va graduellement devenir un élément clef de sa conception de l'histoire. En 1929, Benjamin se réfère encore à l'essai de Lukacs comme l'un des rares livres qui restent vivants et actuels: "Le plus achevé des ouvrages de la littérature marxiste. Sa singularité se fonde sur l'assurance avec laquelle il a saisi d'une part la situation critique de la lutte de classe dans la situation critique de la philosophie, et d'autre part la révolution, désormais concrètement mûre, comme la précondition absolue, voire l'accomplissement et l'achèvement de la connaissance théorique." 2

Ce texte montre quel est l'aspect du marxisme qui intéresse le plus Benjamin et qui va éclairer d'un jour nouveau sa vision du processus historique: la lutte des classes. Mais le matérialisme historique ne va pas se substituer à ses intuitions "anti-progressistes," d'inspiration romantique et messianique: il va s'articuler avec elles, gagnant ainsi une qualité critique qui le distingue radicalement du marxisme "officiel" dominant à l'époque.

Cette articulation se manifeste pour la première fois dans le livre Sens Unique, écrit entre 1923 et 1926, où l'on trouve, sous le titre "Avertisseur d'incendie," cette prémonition historique des menaces 
du progrès: si le renversement de la bourgeoisie par le prolétariat "n'est pas accompli avant un moment presque calculable de l'évolution technique et scientifique (indiqué par l'inflation et la guerre chimique), tout est perdu. II faut couper la mèche qui brûle avant que l'étincelle n'atteigne la dynamite." 3

Contrairement au marxisme évolutioniste vulgaire, Benjamin ne conçoit pas la révolution comme le résultat "naturel" ou "inévitable" du progrès économique et technique (ou de la "contradiction entre forces et rapports de production"), mais comme l'interruption d'une évolution historique conduisant à la catastrophe.

C'est parce qu'il perçoit ce danger catastrophique que Benjamin se réclame, dans son article sur le surréalisme de 1929, du pessimisme - un pessimisme révolutionnaire qui n'a rien à voir avec la résignation fataliste, et encore moins avec le Kulturpessimismus allemand, conservateur, réactionnaire et pré-fasciste (Carl Schmitt, Oswald Spengler, Moeller van der Bruck): le pessimisme est ici au service de l'émancipation des classes opprimées. Sa préoccupation n'est pas le "déclin" des élites, ou de la nation, mais les menaces que fait peser sur l'humanité le progrès technique et économique promu par le capitalisme.

Rien ne semble plus dérisoire aux yeux de Benjamin que l'optimisme des partis bourgeois et de la social-démocratie, dont le programme politique n'est qu'un "mauvais poème de printemps." Contre cet "optimisme sans conscience," cet "optimisme de dilettantes," inspiré par l'idéologie du progrès linéaire, il découvre dans le pessimisme le point de convergence effectif entre surréalisme et communisme..$^{4}$ II va sans dire qu'il ne s'agit pas d'un sentiment contemplatif, mais d'un pessimisme actif, "organisé," pratique, entièrement tendu vers l'objectif d'empêcher, par tous les moyens possibles, l'avènement du pire.

On se demande à quoi peut faire référence le concept de pessimisme appliqué aux communistes: leur doctrine en 1928, célébrant les triomphes de la construction du socialisme en URSS et la chute imminente du capitalisme, n'est-elle précisément un bel exemple d'illusion optimiste? En fait, Benjamin a emprunté le concept d' "organisation du pessimisme" à un ouvrage qu'il qualifie d' "excellent", La Révolution et les intellectuels (1926) du communiste dissident Pierre Naville. Proche des surréalistes (il avait été un des rédacteurs de la revue La Révolution Surréaliste), Naville avait à ce moment fait l'option de l'engagement politique dans le parti communiste français, qu'il veut faire partager à ces amis.

Or, pour Pierre Naville le pessimisme, qui constitue "la source de la méthode révolutionnaire de Marx," est le seul moyen d "'échapper aux nullités et aux déconvenues d'une époque de compromis". Refusant le "grossier optimisme" d'un Herbert Spencer - qu'il gratifie de l'aimable qualificatif de "cervelle monstrueusement rétrécie" - ou d'un Anatole France, dont il insupporte les "infâmes plaisanteries," il conclut: "il faut organiser le pessimisme," "I'organisation du pessimisme" est le seul mot d'ordre qui nous empêche de dépérir. ${ }^{5}$

Inutile de préciser que cette apologie passionnée du pessimisme était très peu représentative de la culture politique du communisme français à cette époque. En fait, Pierre Naville allait bientôt (1928) 
être exclu du Parti: la logique de son anti-optimisme le conduira dans les rangs de l'opposition communiste de gauche ("trotskiste"), dont il deviendra un des principaux dirigeants.

La philosophie pessimiste de l'histoire de Benjamin se manifeste de façon particulièrement aiguë dans sa vision de l'avenir européen: "Pessimisme sur toute la ligne. Oui, certes, et totalement. Méfiance quand au destin de la littérature, méfiance quand au destin de la liberté, méfiance quant au destin de l'homme européen, mais surtout trois fois méfiance en face de tout accommodement: entre les classes, entres les peuples, entre les individus. Et confiance illimitée seulement dans l'I.G. Farben et dans le perfectionnement pacifique de la Luftwaffe." 6

Cette vision critique permet à Benjamin d'apercevoir - intuitivement mais avec une étrange acuité - les catastrophes qui attendaient l'Europe, parfaitement résumées par la phrase ironique sur la "confiance illimitée." Bien entendu, même lui, le plus pessimiste de tous, ne pouvait pas prévoir les destructions que la Luftwaffe allait infliger aux villes et aux populations civiles européennes; et encore moins pouvait-il imaginer que l'I.G. Farben allait, à peine une douzaine d'années plus tard, s'illustrer par la fabrication du gaz ZykIon B utilisé pour "rationaliser" le génocide, ni que ses usines allaient employer, par centaines de milliers, la main d'oeuvre concentrationnaire. Cependant, unique parmi tous les penseurs et dirigeants marxistes de ces années, Benjamin a eu la prémonition des monstrueux désastres dont pouvait accoucher la civilisation industrielle/bourgeoise en crise.

C'est surtout dans le Livre des Passages Parisiens et dans les différents textes des années 1936-40 que Benjamin va développer sa vision de l'histoire, en se dissociant, de façon de plus en plus radicale, des "illusions du progrès" hégémoniques au sein de la pensée de gauche allemande et européenne. Dans un article publié en 1937 dans la célèbre Zeitschrift für Sozialforschung, la revue de l'Ecole de Francfort (déjà exilée aux USA), dédié à l'historien et collectionneur Eduard Fuchs, il s'attaque au marxisme social-démocrate, mélange de positivisme, d'évolutionnisme darwiniste et de culte du progrès: "II ne pouvait voir dans l'évolution de la technique que le progrès des sciences naturelles et pas la régression sociale (...). Les énergies que la technique développe au-delà de ce seuil sont destructrices. Elles mettent en première ligne la technique de la guerre et sa préparation par la presse." 7

L'objectif de Benjamin est d'approfondir et de radicaliser l'opposition entre le marxisme et les philosophies bourgeoises de l'histoire, d'aiguiser son potentiel révolutionnaire et d'élever son contenu critique. C'est dans cet esprit qu'il définit, de façon tranchante, l'ambition du projet des Passages Parisiens: "On peut considérer aussi comme but méthodologiquement poursuivi dans ce travail la possibilité d'un matérialisme historique qui ait annihilé (annihiliert) en lui-même l'idée de progrès. C'est justement en s'opposant aux habitudes de la pensée bourgeoise que le matérialisme historique trouve ses sources." Un tel programme n'impliquait pas un quelconque "révisionisme", mais plutôt, comme Karl Korsch avait tenté de le faire dans son propre livre - une des principales références de Benjamin - un retour à Marx lui-même.

Benjamin était conscient que cette lecture du marxisme plongeait ses racines dans la critique romantique de la civilisation industrielle, mais il était convaincu que Marx lui aussi avait trouvé son inspiration dans cette source. II trouve un appui pour cette interprétation hétérodoxe des origines du marx- 
isme dans le Karl Marx (1938) de Korsch: "Très justement, et non sans nous faire penser à de Maistre et à Bonald, Korsch dit ceci: Ainsi dans la théorie du mouvement ouvrier moderne aussi, il y a une partie de la "désillusion" qui, après la grande Révolution française, fut proclamée par les premiers théoriciens de la contre-révolution et ensuite par les romantiques allemands et qui, grâce à Hegel, eut une forte influence sur Marx." 9

La formulation la plus étonnante et radicale de la nouvelle philosophie de l'histoire - marxiste et messianique - de Walter Benjamin se trouve sans doute dans les Thèses Sur le concept d'histoire de 1940, un des documents les plus importants de la pensée révolutionnaire depuis les Thèses sur Feuerbach de 1845.

L'exigence fondamentale de Benjamin c'est d'écrire l'histoire "à rebrousse poil," c'est à dire du point de vue des vaincus - contre la tradition conformiste de l'historicisme allemand dont les partisans entrent toujours "en empathie avec le vainqueur" (Thèse VII). ${ }^{10}$

\| va de soi que le mot "vainqueur" ne fait pas référence aux batailles ou aux guerres habituelles, mais à la "guerre des classes" dans laquelle l'un des camps, la classe dirigeante, "n’a pas cessé de l'emporter" (Thèse VII) sur les opprimés - depuis Spartacus, le gladiateur rebelle, jusqu'au groupe Spartacus de Rosa Luxemburg, et depuis l'Imperium romain jusqu'au Tertium Imperium nazi. L'historicisme s'identifie empathiquement (Einfühlung) aux classes dominantes. II voit l'histoire comme une succession glorieuse de hauts faits politiques et militaires. En faisant l'éloge des dirigeants et en leur rendant hommage, il leur confère le statut d'"héritiers" de l'histoire passée. En d'autres termes, il participe - comme ces personnages qui élèvent la couronne de lauriers au dessus de la tête du vainqueur - à "ce cortège triomphal où les maîtres d'aujourd'hui marchent sur les corps des vaincus" (Thèse VII).

La critique que Benjamin formule à l'encontre de l'historicisme s'inspire de la philosophie marxiste de I'histoire, mais elle a aussi une origine nietzschéenne. Dans une de ses oeuvres de jeunesse, De l'utilité et de l'inconvénient de l'histoire (citée dans la Thèse XII), Nietzsche tourne en ridicule l' "admiration nue du succès" des historicistes, leur "idolâtrie pour le factuel" (Götzerdienste des Tatsächlichen) et leur tendance à s'incliner devant la "puissance de l'histoire." Puisque le Diable est le maître du succès et du progrès, la véritable vertu consiste à se dresser contre la tyrannie de la réalité et à nager contre le courant historique.

II existe un lien évident entre ce pamphlet nietzschéen et l'exhortation de Benjamin à écrire l'histoire gegen den Strich. Mais les différences ne sont pas moins importantes: alors que la critique de Nietzsche contre l'historicisme se fait au nom de la "Vie" ou de I'"Individu héroïque," celle de Benjamin parle au nom des vaincus. En tant que marxiste, ce dernier se situe aux antipodes de l'élitisme aristocratique du premier et choisit de s'identifier avec les "damnés de la terre," ceux qui sont couchés sous les roues de ces chars majestueux et magnifiques appelés Civilisation ou Progrès.

Rejetant le culte moderne de la Déesse Progrès, Benjamin met au centre de sa philosophie de l'histoire le concept de catastrophe. Dans une des notes préparatoires aux Thèses de 1940 il observe: "La catastrophe est le progrès, le progrès est la catastrophe. La catastrophe est le continuum de I'histoire." 11 L'assimilation entre progrès et catastrophe a tout d'abord une signification historique: 
le passé n'est, du point de vue des opprimés, qu'une série interminable de défaites catastrophiques. La révolte des esclaves, la guerre des paysans, juin 1848, la Commune de Paris, le soulèvement berlinois de janvier 1919 - ce sont des exemples qui apparaissent souvent dans les écrits de Benjamin, pour lequel "cet ennemi n'a pas cessé de vaincre" (Thèse VI). Mais cette équation a aussi une signification éminemment actuelle, parce que, "à l'heure qu'il est, l'ennemi n'a pas encore fini de triompher" (Thèse VI, traduction de Benjamin lui-même en français): défaite de l'Espagne républicaine, Pacte Molotov-Ribbentrop, invasion nazie victorieuse en Europe.

Le fascisme occupe bien évidemment une place centrale dans la réflexion historique de Benjamin dans les Thèses. Pour lui ce n'est pas un accident de l'histoire, un "état d'exception," quelque chose d'impossible au XXème siècle, une absurdité du point de vue du progrès: rejettant ce type d'illusion, Benjamin appelle de ses voeux "une théorie de l'histoire à partir de laquelle le fascisme puisse être perçu," ${ }^{12}$ c'est à dire une théorie qui comprenne que les irrationalités du fascisme ne sont que l'envers de la rationalité instrumentale moderne. Le fascisme porte à ses dernières conséquences la combinaison typiquement moderne entre progrès technique et régression social.

Tandis que Marx et Engels avaient eu, selon Benjamin, "l'intuition fulgurante" de la barbarie à venir dans leur pronostic sur l'évolution du capitalisme, ${ }^{13}$ leurs épigones du XXème siècle ont été incapables de comprendre - et donc de lui résister efficacement - une barbarie moderne, industrielle, dynamique, installée au coeur même du progrès technique et scientifique.

Cherchant les racines, les fondements méthodo-logiques de cette incompréhension catastrophique, qui a contribué à la défaite du mouvement ouvrier allemand en 1933, Benjamin s'attaque à l'idéologie du progrès dans toutes ses composantes: l'évolutionnisme darwiniste, le déterminisme de type scientifico-naturel, l'optimisme aveugle - dogme de la victoire "inévitable" du parti - la conviction de "nager dans le sens du courant" (le développement technique); en un mot, la croyance confortable dans un progrès automatique, continu, infini, fondé sur l'accumulation quantitative, l'essor des forces productives et l'accroissement de la domination sur la nature. II croit déceler derrière ces manifestations multiples un fil conducteur qu'il soumet à une critique radicale: la conception homogène, vide et mécanique (comme un mouvement d'horlogerie) du temps historique.

Contre cette vision linéaire et quantitative, Benjamin oppose une perception qualitative de la temporalité, fondée d'une part sur la remémoration, de l'autre sur la rupture messianique/révolutionnaire de la continuité. La révolution est le "correspondant" (au sens baudelairien du mot) profane de l'interruption messianique de I'histoire, de l'"arrêt messianique du devenir" (Thèse XVII): les classes révolutionnaires, écrit la Thèse XV, sont conscientes, au moment de leur action, de "briser le continuum de l'histoire." L'interruption révolutionnaire est donc la réponse de Benjamin aux menaces que fait peser sur l'espèce humaine la poursuite de la tempête maléfique qu'on appelle "Progrès," une tempête qui accumule ruine sur ruine et prépare des catastrophes nouvelles (Thèse XII). C'était I'année 1940, peu avant Auschwitz et Hiroshima...

Selon Habermas, il existe une contradiction entre la philosophie de l'histoire de Benjamin et le matérialisme historique. L'erreur de Benjamin fut selon lui d'avoir voulu imposer - "comme un capuchon de 
moine sur la tête" - au matérialisme historique marxien, "qui tient compte des progrès non seulement dans le domaine des forces productives mais aussi celui de la domination," "une conception historique anti-évolutionniste." ${ }^{14}$

En réalité, une interprétation dialectique et non-évolutionniste de l'histoire, prenant en compte à la fois les progrès et les régressions - comme l'ont fait Benjamin et ses amis de l'Ecole de Francfort - peut se fonder sur plusieurs écrits de Marx. II est cependant vrai qu'elle entre en conflit avec les interprétations dominantes du matérialisme historique développées au cours du XXème siècle. Ce qu'Habermas pense être une erreur est précisément la source de la valeur singulière de la philosophie benjaminienne de l'histoire, et de sa capacité à comprendre un siècle caractérisé par l'imbrication étroite de la modernité et de la barbarie.

\section{(Ed. Rika Benveniste)}

${ }^{1}$ W.Benjamin, "La vie des étudiants", 1915, dans Mythe et Violence, Lettres Nouvelles, 1971, p. 37.

${ }^{2}$ W.Benjamin, Gesammelte Schriften, Francfort, Suhrkamp Verlag, 1980, III, p. 171.

${ }^{3}$ W.Benjamin, Sens Unique, Paris, Lettres Nouvelles / Maurice Nadeau, 1978, pp. 205-206.

${ }^{4}$ W.Benjamin, "Le surréalisme. Le dernier instantané de l'intelligence européenne”, Mythe et Violence, p. 312.

${ }^{5}$ Pierre Naville, La révolution et les intellectuels, Paris, Gallimard, 1965 , pp. 76-77, 110-117.

${ }^{6}$ W.Benjamin, “Le surréalisme”, p. 312.

${ }^{7}$ W.Benjamin, Gesammelte Schriften, III, p. 474.

${ }^{8}$ W.Benjamin, Passagenwerk - Gesammelte Schritten V, p. 574.

${ }^{9}$ W.Benjamin, Ibid., p. 820.

${ }^{10}$ Les citations des "Thèses sur la philosophie de l'histoire" sont le plus souvent tirées de la traduction de Maurice de Gandillac dans Poésie et Révolution, Paris, Lettres Nouvelles, 1971.

${ }^{11}$ W.Benjamin, G.Schriften, I, 3, p. 1244 (notes préparatoires pour les Thèses).

${ }^{12}$ W.Benjamin, G.S., I, 3, p.1244 (notes préparatoires).

${ }^{13}$ W.Benjamin, G.S., II, 2, p. 488.

${ }^{14}$ J.Habermas, "L'actualité de W. Benjamin. La critique: prise de conscience ou preservation", Revue d'esthétique ${ }^{\circ} 1$, 1981, p. 121. 